UDC: 519.63

\title{
Application of the grid-characteristic method for mathematical modeling in dynamical problems of deformable solid mechanics
}

\author{
I. B. Petrov \\ Moscow Institute of Physics and Technology, \\ 9 Institutskii per., Dolgoprudny, Russia \\ E-mail: petrov@mipt.ru
}

Received 24.05.2019.

Accepted for publication 14.11.2019.

\begin{abstract}
The grid-characteristic method is a promising numerical method for solving hyperbolic systems of equations, e.g., equations describing elastic and acoustic waves. This method has high precision and allows physically correct simulations of wave processes in heterogeneous media. The grid-characteristic method makes it possible to correctly take into account boundary conditions and conditions on surfaces with different physical characteristics. The method offers the greatest advantages for one-dimensional equations, especially in combination with a fixed difference grid, as in conventional grid-based methods. However, in the multidimensional case using the algorithms of splitting with respect to spatial variables, the author has managed to preserve its positive qualities. The use of the method of Runge-Kutta type, or the integro-interpolation method for hyperbolic equations makes it possible to effectively carry out a generalization of methods developed for linear equations, in the nonlinear case, in particular, to enforce the difference analogs of the conservation laws, which is important for shock-capturing, for example, discontinuous solutions. Based on the author's variant of the grid-characteristic method, several important problems of seismic prospecting, seismic resistance, global seismic studies on Earth and Mars, medical applications, nondestructive testing of railway lines, the simulation of the creation and characteristics of composite materials for the aerospace industry and other areas of practical application were numerically solved. A significant advantage of the constructed method is the preservation of its stability and precision at the strains of the environment. This article presents the results of a numerical solution based on the grid-characteristic method to the problem of modeling elastic-plastic deformation in traumatic brain injury.
\end{abstract}

Keywords: hyperbolic type equations, elastic and plastic deformation, grid-characteristic method, finite difference schemes, discontinuous solutions

Citation: Computer Research and Modeling, 2019, vol. 11, no. 6, pp. 1041-1048.

This work was supported by the Russian Science Foundation, project no. 19-11-00023. 


\section{Introduction}

The numerical solution to nonstationary dynamic problems of elastic and elastic-plastic deformations of isotropic solids in the case of two or three spatial coordinates is the object of many works, a fairly detailed overview is given in [Kukudzhanov, 1976; Novatskii, 1978]. In solid mechanics various mathematical rheological models have been developed. Among these models, special mention should be made of those which are described by systems of equations of hyperbolic type, and, above all, the models of linear and nonlinear theory of elasticity and plasticity.

The most important concept for such equations is their characteristic properties. The use of the characteristic records of the original equations when constructing a particular numerical method (i.e., approximation of not the original equations, but equivalent conditions of compatibility along some characteristic directions) allows in the most natural way building a computational algorithm on the boundaries of the region of integration, to a certain extent taking into account the solutions dependence domain, etc. The advantages of the characteristic approaches show themselves particularly for onedimensional equations, especially in combination with a fixed difference grid, as in conventional gridbased methods (grid-characteristic methods [Magomedov, Kholodov, 1969; Kholodov, 1978; Kholodov, 1980]; however, in the multidimensional case, when the algorithms of spatial splitting are used, the variables are able to preserve their positive qualities. The use of the equations of Runge-Kutta type or the integro-interpolation method [Rusanov, 1968; Tikhonov, Samarskiy, 1961] makes it possible to effectively generalize methods developed for linear equations, in the nonlinear case, in particular, by ensuring the implementation of difference analogs of the conservation laws, which is important for shock capturing of discontinuous solutions.

Among other approaches widely used for numerical solution to such problems, we will note the method of disintegration of discontinuities and various difference schemes of splitting. The choice of the coordinate system used is crucial at considerable strains of bodies. If for small displacements (and strains) commonly encountered in solid mechanics problems, the Lagrangian finite-difference grid undergoes no significant changes, then with large deformations in the area of integration heavy distortions on it are possible until disturbances arise (zero area of differential cells, their "eversion", etc.). Methods used in such cases for regularization of difference grids (reinterpolation on a new grid, etc.) do not always give the expected effect.

On the other hand, the use of space-fixed Eulerian coordinates leads to serious difficulties in the approximation of boundary conditions. Therefore, it is important to compare calculations performed in various coordinate systems, using the same numerical scheme that is one of the objectives of the present work.

\section{Mathematical model}

Using conventional kinematic equations for a symmetric tensor of velocities of deformations in the space-fixed orthogonal curvilinear coordinate system $x_{1}, x_{2}$ and $x_{3}$

$$
\begin{gathered}
e_{m n}=\frac{1}{2}\left(\frac{1}{H_{n}} \frac{\partial v_{m}}{\partial x_{n}}+\frac{1}{H_{m}} \frac{\partial v_{n}}{\partial x_{m}}\right)+\frac{1}{H_{n}}\left[\delta_{m n}\left(\sum_{s=1}^{3} \frac{v_{s}}{H_{s}} \frac{\partial H_{m}}{\partial x_{s}}\right)-\frac{1}{2 H_{m}}\left(v_{m} \frac{\partial H_{m}}{\partial x_{n}}+\frac{\partial H_{n}}{\partial x_{m}}\right)\right], \\
m, n=1,2,3 .
\end{gathered}
$$

choosing the defining relations in the form

$$
\frac{d \sigma_{i j}}{d t}=\frac{\partial \sigma_{i j}}{\partial t}+\sum_{k=1}^{3}\left(\frac{v_{k}}{H_{k}} \frac{\partial \sigma_{i j}}{\partial x_{k}}\right)
$$


we write a closed system of two-dimensional nonstationary equations in the form

$$
\frac{\partial \vec{u}}{\partial t}+\sum_{i=1,2} \mathbf{A}_{\mathbf{i}} \frac{\partial \vec{u}}{\partial \xi_{i}}=\vec{f} .
$$

The symbol $\vec{u}=\left\{V_{1}, V_{2}, \sigma_{11}, \sigma_{12}, \sigma_{22}, \sigma_{33}, T\right\}$ means the vector of desired variables, including the components $v_{1}$ and $v_{2}$ of the velocity vector $V$ (on the axes $x_{1}, x_{2}$, respectively), nonzero components $\sigma_{i j}$ of the symmetric stress tensor and the temperature $T$; $\vec{f}\left(t, x_{1}, x_{2}, \vec{u}\right)=$ $=\left(f_{1}, f_{2}, f_{11}, f_{22}, f_{33}, f_{T}\right)$ - the vector of the right parts with the following components in the curvilinear orthogonal coordinate system $x_{1}, \ldots 2, \ldots 3$

$$
\begin{aligned}
f_{1} & =F_{1}+\frac{1}{\rho}\left[\frac{\left(\sigma_{11}-\sigma_{22}\right)}{H_{1} H_{2}}+\frac{\partial H_{2}}{\partial x_{1}}+\frac{\left(\sigma_{11}-\sigma_{33}\right)}{H_{1} H_{3}}+\frac{\sigma_{12}}{H_{2}}\left(\frac{2}{H_{1}} \frac{\partial H_{1}}{\partial x_{2}}+\frac{1}{H_{3}} \frac{\partial H_{3}}{\partial x_{2}}\right)\right]- \\
& -\frac{v_{2}}{H_{1} H_{2}}\left(v_{1} \frac{\partial H_{1}}{\partial x_{2}}+v_{2} \frac{\partial H_{2}}{\partial x_{1}}\right), \\
f_{2} & =F_{2}+\frac{1}{\rho}\left[\frac{\left(\sigma_{22}-\sigma_{33}\right)}{H_{2} H_{3}}+\frac{\partial H_{3}}{\partial x_{2}}+\frac{\left(\sigma_{22}-\sigma_{11}\right)}{H_{2} H_{1}}+\frac{\sigma_{12}}{H_{1}}\left(\frac{2}{H_{2}} \frac{\partial H_{2}}{\partial x_{1}}+\frac{1}{H_{3}} \frac{\partial H_{3}}{\partial x_{1}}\right)\right]- \\
& -\frac{v_{1}}{H_{2} H_{1}}\left(v_{2} \frac{\partial H_{2}}{\partial x_{1}}+v_{1} \frac{\partial H_{1}}{\partial x_{2}}\right), \\
f_{i j} & =\frac{q_{i j 11} v_{2}}{H_{1} H_{2}} \frac{\partial H_{2}}{\partial x_{2}}-\frac{q_{i j 1}-q_{i j 21}}{2 H_{1} H_{2}}\left(v_{1} \frac{\partial H_{1}}{\partial x_{2}}+v_{2} \frac{\partial H_{2}}{\partial x_{1}}\right)+ \\
& +\frac{q_{i j 22} v_{2}}{H_{1} H_{2}} \frac{\partial H_{2}}{\partial x_{1}}+\frac{q_{i j 33}}{H_{3}}\left(\frac{v_{1}}{H_{1}} \frac{\partial H_{3}}{\partial x_{1}}+\frac{v_{2}}{H_{2}} \frac{\partial H_{3}}{\partial x_{2}}\right)-\frac{\delta_{i j} \gamma Q}{\rho c} \\
f_{T} & =\frac{1}{\rho c}\left[\frac{\sigma_{11} v_{2}}{H_{1} H_{2}} \frac{\partial H_{1}}{\partial x_{2}}-\frac{\sigma_{22}}{H_{1} H_{2}}+\left(v_{1} \frac{\partial H_{1}}{\partial x_{2}}+v_{2} \frac{\partial H_{2}}{\partial x_{1}}\right)+\right. \\
& \left.+\frac{\sigma_{22} v_{1}}{H_{1} H_{2}} \frac{\partial H_{2}}{\partial x_{1}}+\frac{\sigma_{33}}{H_{3}}\left(\frac{v_{1}}{H_{1}} \frac{\partial H_{3}}{\partial x_{1}}+\frac{v_{2}}{H_{2}} \frac{\partial H_{3}}{\partial x_{2}}\right)+Q\right] .
\end{aligned}
$$

Here $F_{1}, F_{2}$ are the components of the vector of mass forces, $\gamma$ is the internal energy for the thermoelastic medium, $c$ is the specific heat of the material, $T$ is the temperature, $Q$ is the density of heat sources; $H_{i}, i=1,2,3$ are the Lame coefficients that characterize the selectable orthogonal curvilinear coordinate system, $\rho$ is the density determined by the equation of state of a rigid body.

\section{Results of numerical modeling}

\section{Traumatic brain injuries}

This task was set by specialists of the Sklifosovsky Institute and the Burdenko Ambulance Central Clinical Military Hospital.

Working together with the neurosurgeons, we formulated three mechanical-mathematical models of human head. The simplest of these is a two-component model (Figure 1a), in which bone tissue and brain are described by homogeneous isotropic materials with average mechanical properties; more complex models take into account the presence of ventricle (Figure 1b) and two membranes (Figure 1c).

The external load is set as a collision of the skull-brain system with a completely fixed rigid barrier with a given initial velocity $(1-3 \mathrm{~m} / \mathrm{s})$. It is known that heterogeneity makes a significant contribution to the formation of a wave pattern of disturbance propagation in an elastic medium. Therefore, a two-component model was further developed in the form of a three-component model, consisting of bones and brain and ventricle. The dural membranes have an inhibiting effect on the 


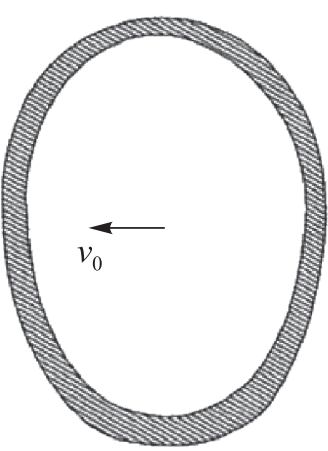

(a)

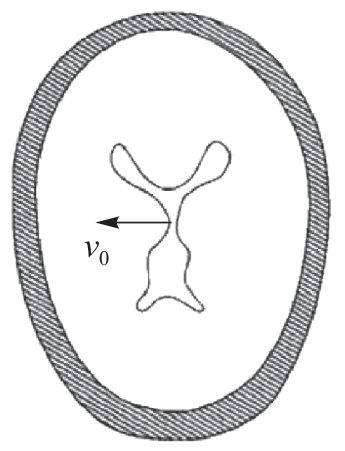

(b)

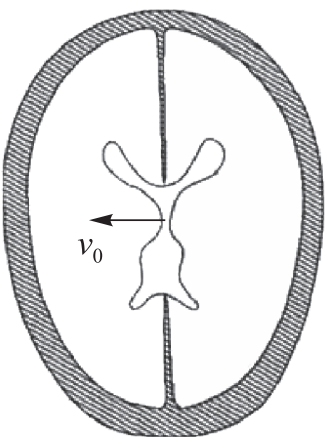

(c)

Figure 1. Mechanical-mathematical models of human head

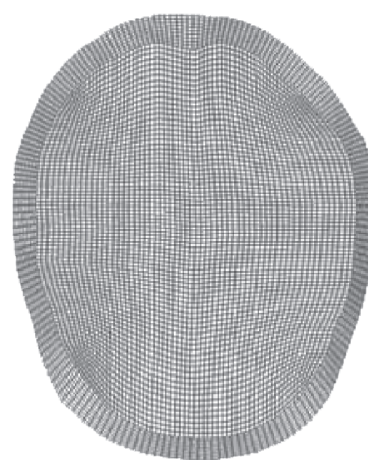

(a)

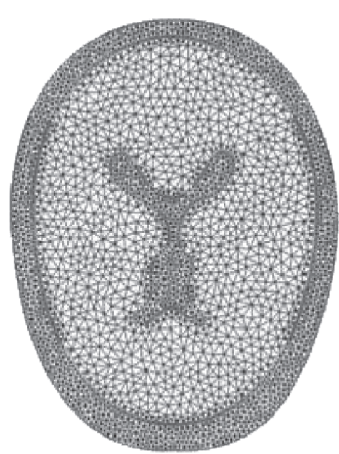

(b)

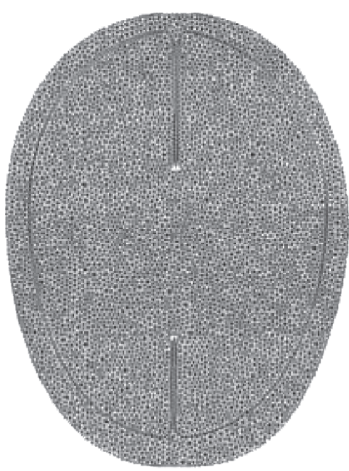

(c)

Figure 2. Computational grids for describing models

movement of brain inside skull. Therefore, subsequently, it was decided to introduce a model of falx cerebri, with a vertical membrane separating the hemispheres in the parietal region. Figure 2 shows the corresponding computational grids ( $a$ - quadrangular, $b$ and $c$ - triangular) for these models, which were based on numerical calculations. Rheological properties of biomaterials were also varied. Thus, the rheology of the medulla varied from lianopoulos to viscoelastic.

The behavior of bone material was simulated by lianopoulos continuous medium with average properties of lamellar and trabecular bone. Modeling the interaction of skull-brain is challenging due to the fact that brain has several different mechanical properties of the membranes with cavities, including fluid-filled ones. This work addresses the selection of the contact gap with conditions that ranged from full adhesion to slip with the possibility of detachment.

The distribution of the shear stress in the formulation of various conditions is shown in Figures 3 and 4. The model of traumatic brain sections: $a$ - a two-component model, $b$ - a model of ventricles, $c-$ a model with ventricles and a membrane (falx cerebri).

Figure 3 shows the overall characteristics of the mechanical effects on the brain at a side impact, obtained using different models with free-slip on the border of the skull-brain. The most dangerous are concentrations of maximum tensile (positive) and shear stress. The use of conditions for the complete adhesion on the border of the skull-brain leads to concentration of the shear stress along the contact boundary on the lateral surfaces, while the sliding contact completely removes them. Accounting for the presence of ventricles has almost no effect on the distribution of the regions of maximum compression and extension, but significantly affects the distribution of shear loads. The presence of membrane is more essential to localize areas of compression-tension in side impacts. Figure 5a shows an example 


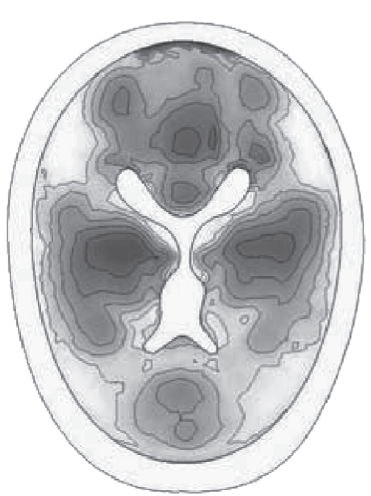

(a)

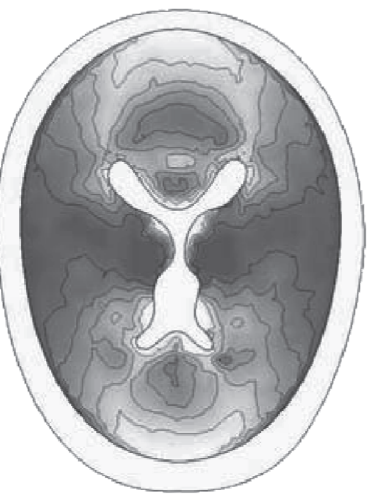

(b)

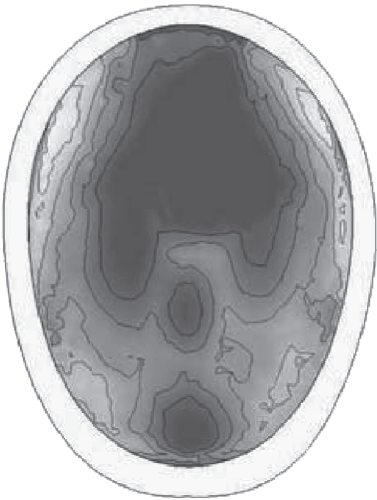

(c)

Figure 3. The distribution of shear stresses: $\mathrm{a}$ - complete adhesion; $\mathrm{b}-$ slide; $\mathrm{c}-$ two-component model
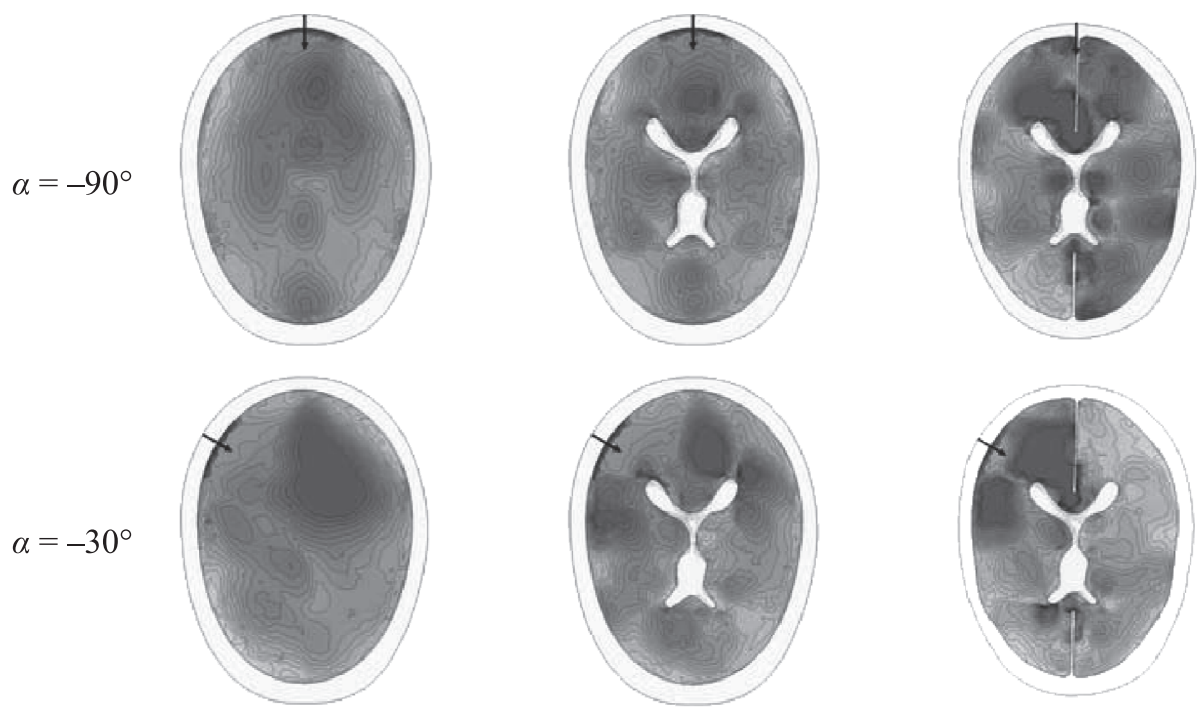

Figure 4. The dependence of the distribution of the maximum shear stresses $(0 \div 0.04$ ATM $)$ on the impact direction (dark regions correspond to larger shears): $\mathrm{a}-\mathrm{a}$ two-component model; $\mathrm{b}-\mathrm{a}$ model with ventricles; c - a model with a membrane

of a CT image of a patient with brain contusion of severe degree with left impact from an accident (data provided by the Burdenko Main Military Hospital). The arrows on the CT scan indicate the lesion of the brain substance. Figure $5 \mathrm{~b}$ shows the corresponding distribution of maximum shear stress. When reducing the area of impact (similar to impact with a pointed object) there is a region of concentration of the shear stress, which coincides with the center of hematoma.

Figure 4 shows the distribution fields of shear stress in human brain depending on the angle of application of the shock load for the three models of human head, which gives the neurosurgeon information about the localization and volume of the region of brain damage in traumatic brain injury.

\section{Direct problems of exploration seismology}

Figure 6 shows the isosurfaces of the modulus of the velocity for ten-layer geological environment at four points in time effects of seismic loading at the upper boundary.

Let us consider the problem of reflection of plane Ricker pulse, the direction of propagation of which is 14 degrees with vertical (this corresponds to the displacement of the point of the explosion 


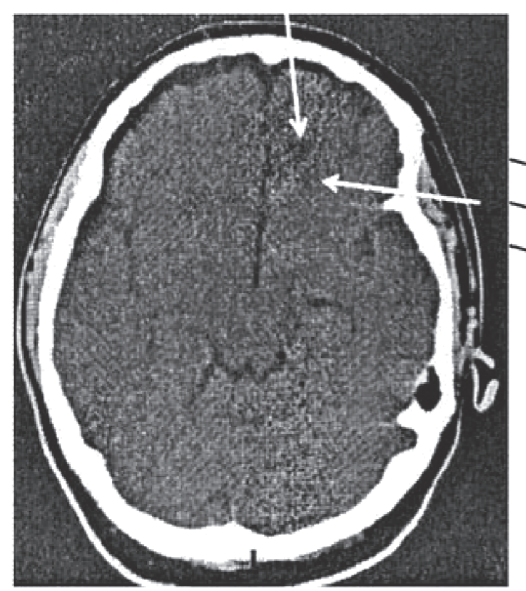

(a)

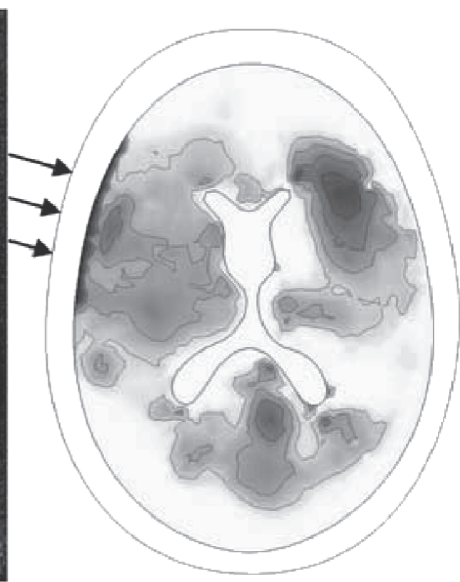

(b)

Figure 5. $a-\mathrm{CT}$ of a patient with brain contusion of severe degree, left impact; $b$ - the corresponding distribution of maximum shear stress

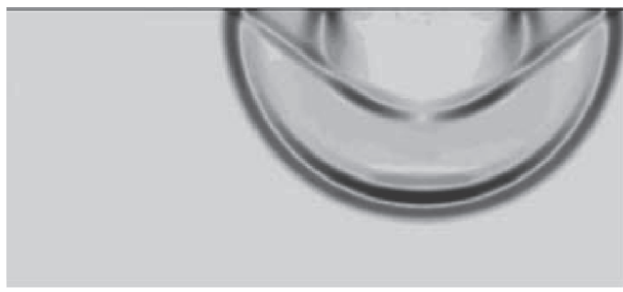

(a)

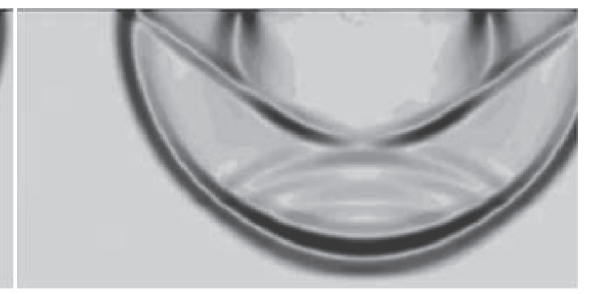

(b)

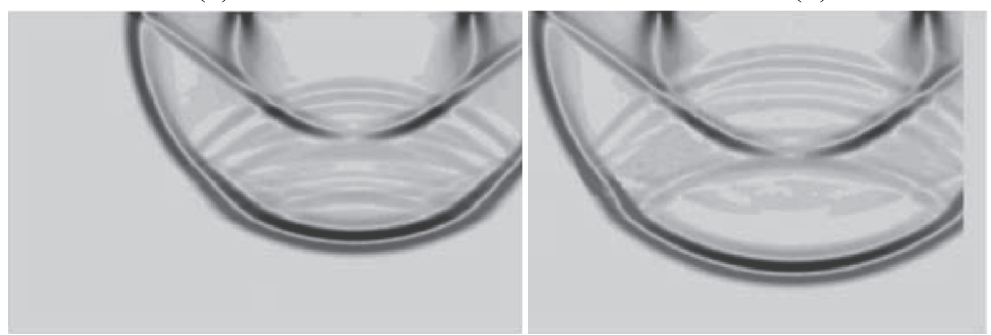

(c)

(d)

Figure 6. The isosurfaces of the modulus of the velocity for ten-layer geological environment at four points in time (see Figure a, b, c, d for $500 \mathrm{~ms}, 600 \mathrm{~ms}, 700 \mathrm{~ms}, 800 \mathrm{~ms}$, respectively)

along the surface to $0.5 \mathrm{~km}$ ) from the reservoir 5 vertical filled cracks [Kvasov et al., 1961]. The pattern of reflected waves at various distances between the cracks is examined. The q value will change from 0.5 to $4.0(q=0.5,1.0,1.5,2.0,3.0,4.0)$. Figure 7 shows the corresponding velocity fields.

A comparison is made between the results of calculations of the geological problems on the two-dimensional triangular grid on a three-dimensional cubic grid. This comparison shows that the discrepancy of the results obtained is not more than $30 \%$. Thus, the two-dimensional calculation gives not only a qualitatively correct result, but also a good quantitative assessment. Therefore, in the study of three-dimensional dynamic processes in geological environments it is possible to use two-dimensional calculations. This can significantly speed up computation time and reduce storage costs and data processing.

It is seen that the numerical scheme used in this work gives a good complex wave pattern resulting from the interaction of seismic waves with a geological layered rock. On the calculated seismogram the waves reflected from all contact boundaries are clearly visible. The calculations 


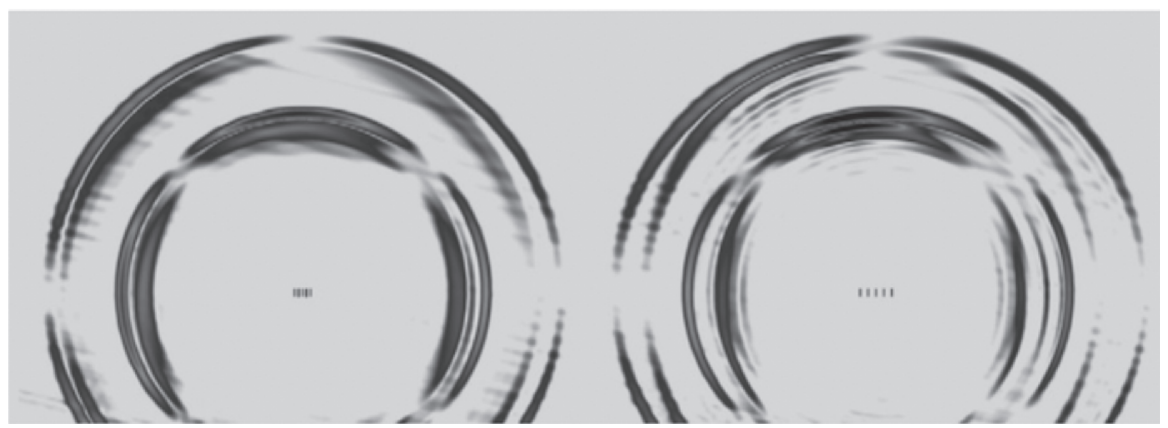

(a)

(b)

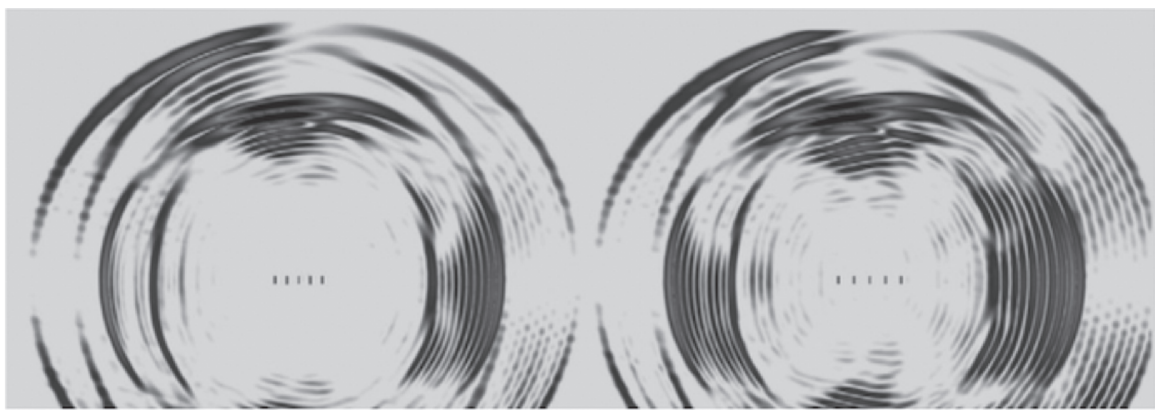

(c)

(d)

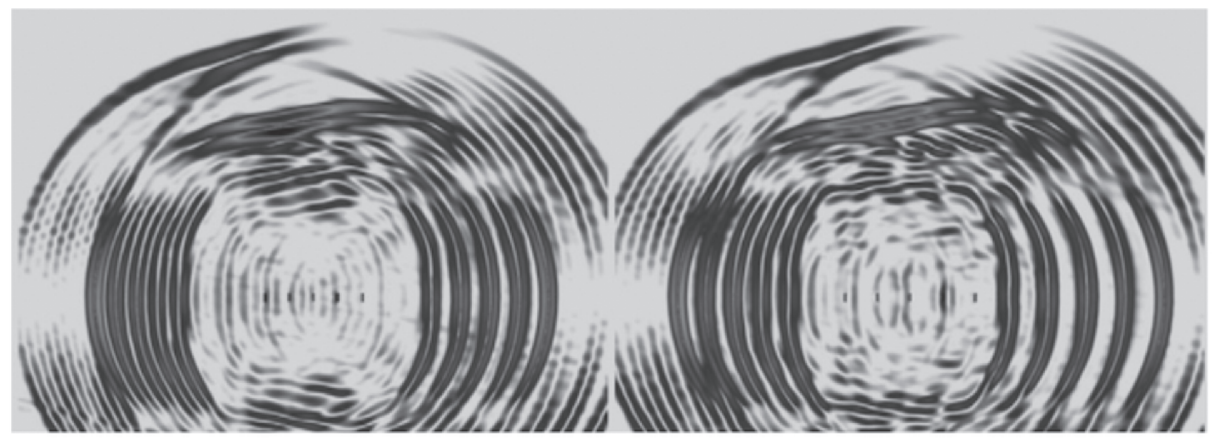

(e)

(f)

Figure 7. Velocity fields from fractures

obtained show the possibility of using a full numerical simulation of complex wave processes in layered geological media to obtain data and to study the structure of rocks.

\section{Impact task}

Similar designs are used as protective ones (e.g., body armor). The lower (in the figures on the right) calculated layer corresponds to the human body protected from hitting [Petrov et al., 2016]. As can be seen from the calculations, after hitting the upper boundary of the five-layer construction, the effect of elastic-plastic compression waves leads to a collision of layers from top to bottom, after which the compression waves go to a protected environment. By simulating attacks on the multilayer barrier we obtained a wave pattern corresponding to the distribution of the secondary waves generated by the collisions of obstacles and directed against the impact. In addition, the layers in such structures then go away from each other, depending on the sign of the normal stress (negative or positive) on the contact boundaries. These processes (collision and detachment) initiate the emergence of secondary waves of compression-stretching in the design and the protected body (Figure 8). 


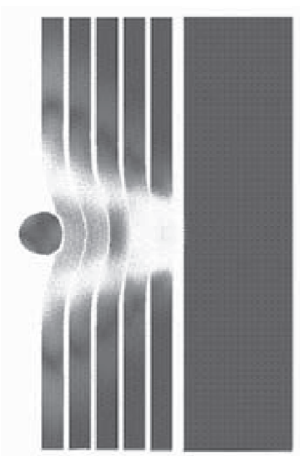

(a)

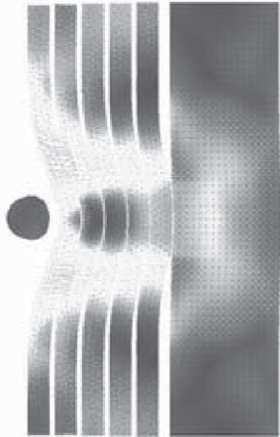

(b)

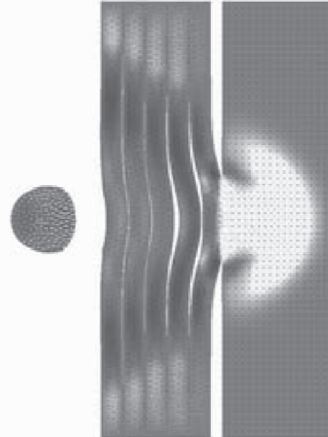

(c)

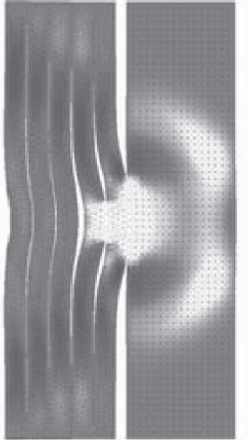

(d)

Figure 8. Results of calculations (velocity fields) for the impacts on five-layer barriers with gaps (spaced obstacles) and without gaps between layers

\section{References}

Kholodov A.S. O postroenii raznostnykh skhem s polozhitel'noy approksimatsiey dlya uravneniy giperbolicheskogo tipa [On construction of difference schemes with positive approximation for equations of hyperbolic type] // Zh. vychisl. matem. i matem. fiz. - 1978. - Vol. 18, No. 6. P. 1476-1492 (in Russian).

Kholodov A.S. O postroenii raznostnykh skhem povyshennogo poryadka tochnosti dlya uravneniy giperbolicheskogo tipa [On construction of difference schemes of increased order of accuracy for equations of hyperbolic type] // Zh. vychisl. matem. i matem. fiz. -1980 . - Vol. 20, No. 6. P. 1601-1620 (in Russian).

Kukudzhanov V.N. Chislennoe reshenie neodnomernykh zadach rasprostraneniya voln napryazheniy $\mathrm{v}$ tverdykh telakh [Numerical solution of multidimensional problems of propagation of stress waves in solid bodies] // Soobshch. po prikl. matem. - Iss. 6. - Moscow, VTS AN USSR, 1976. - 65 p. (in Russian).

Kvasov I. E., Leviant V. B., Petrov I. B. Chislennoe issledovanie volnovykh protsessov v poristoy srede $\mathrm{s}$ ispol'zovaniem setochno-kharakteristicheskogo metoda [Numerical study of wave processes in a porous medium using grid-characteristic method] // Zh. vychisl. matem. i matem. fiz. - 2016. Vol. 56, No. 9. - P. 1645-1656 (in Russian).

Magomedov K. M., Kholodov A.S. O postroenii raznostnykh skhem dlya uravneniy giperbolicheskogo tipa na osnove kharakteristicheskikh sootnosheniy [On construction of difference schemes for equations of hyperbolic type on the basis of the characteristic ratios] // $\mathrm{Zh}$. vychisl. matem. i matem. fiz. - 1969. - Vol. 9, No. 2. - P. 373-386 (in Russian).

Novatskii V.K. Volnovye zadachi teorii plastichnosti [Wave problems of the theory of plasticity]. Moscow: Mir, 1978. - 307 p. (in Russian).

Petrov I., Vasyukov A., Ermakov A., Favorskaya A. Numerical modeling of non-destructive testing of composities // Science Processing Computer Science. - 2016. - No. 96. - P. 930-938.

Rusanov V.V. Raznostnye skhemy tret'ego poryadka tochnosti dlya skvoznogo scheta razruvnykh resheniy [Difference schemes of third order of accuracy for calculation of discontinuous solutions] // Dokl. AN USSR. - 1968. - Vol. 180, No. 6. - P. 1303-1305 (in Russian).

Tikhonov A.I., Samarskiy A.A. Ob odnorodnykh raznostnykh skhemakh [On homogeneous difference schemes] // Zh. vychisl. matem. i matem. fiz. - 1961. - Vol. 1, No. 1. - P. 5-63 (in Russian). 